\title{
Diluent Segregation in Crystalline/Amorphous Poly(vinylidene fluoride)/Poly(vinyl acetate) Blends. Segregation Distance Dominated by the Crystal Growth Kinetics
}

\author{
Hsin-Lung Chen, ${ }^{\dagger}$ Shu-Fen Wu, Tsang-Lang Lin, ${ }^{*}$ and Gwo-Mei Wu** \\ Department of Chemical Engineering, National Tsing Hua University, \\ Hsin-Chu 30013, Taiwan, Republic of China \\ ${ }^{*}$ Department of Engineering and System Science, National Tsing Hua University, \\ Hsin-Chu 30013, Taiwan, Republic of China \\ ** Department of Chemical and Materials Engineering, Chang Gung University, \\ Kwei-San, Taoyuan 333, Taiwan, Republic of China
}

(Received November 26, 2001; Accepted March 5, 2002)

\begin{abstract}
We systematically studied the effects of composition and crystallization temperature $\left(T_{\mathrm{c}}\right)$ on the morphological patterns of poly(vinylidene fluoride) (PVDF)/poly(vinyl acetate) (PVAc) blends by means of small angle X-Ray scattering (SAXS). The absolute intensity was measured so that the morphological structures can be resolved from the intensity perturbation. The scattering intensity associated with the PVDF lamellar stacks was strongly enhanced upon blending with PVAc. This was due to the presence of amorphous PVAc in the interlamellar regions that enhanced the electron density contrast between the crystalline and amorphous layers. Except for an intermediate $T_{\mathrm{c}}$ of $110^{\circ} \mathrm{C}$ where the systems attained the maximum crystal growth rate, partial extralamellar segregation of PVAc became prevalent when the PVAc composition exceeded 20 or $30 \mathrm{wt} \%$. Even crystallization at as low as $20^{\circ} \mathrm{C}$ (where the diluent mobility was expected to be sluggish) produced a large extent of extralamellar segregation due to slow crystal growth. The results suggested that the crystal growth kinetics has dominated over the diluent mobility in controlling the average distance of diluent segregation in PVDF/PVAc with the prescribed molecular weight combination.

KEY WORDS Blends / Crystallization / Diluent Segregation / Morphology / Crystal Growth Kinetics /
\end{abstract}

Melt-miscible crystalline/amorphous (C/A) blends continue to receive attentions due to their rich morphology generated through the crystallization process. When liquid-liquid phase separation is absent below the melting point, crystallization in C/A blends usually involves two types of polymer transport, namely, diffusion of the crystalline component towards the crystal growth front and a simultaneous segregation of the amorphous diluent away from the growth front. ${ }^{1}$ Such a liquid-solid phase separation may create a variety of morphological patterns characterized by the location of the diluent, where the following three types have typically been recognized: ${ }^{2}$

(1) Interlamellar (IL) segregation, where the diluent is expelled by a short distance (several $\mathrm{nm}$ ) such that it is incorporated into the amorphous regions between the individual lamellae.

(2) Interfibrillar (IF) segregation, where the diluent is segregated by a larger distance (tens of $\mathrm{nm}$ to $\mu \mathrm{m}$ ) to the regions between the lamellar bundles in the spherulites.

(3) Interspherulitic (IS) segregation, where the diluent is rejected by the longest distance such that it is not contained within the spherulites.

${ }^{\dagger}$ To whom correspondence should be addressed.
The later two types characterized by the longer segregation distance are also termed as "extralamellar segregations". A blend system does not necessarily exhibit only one type of morphology; different morphological patterns may coexist owing to multiple locations of the amorphous diluent. ${ }^{3-6}$

Although the morphology of C/A blends has been widely investigated, the controlling factors associated with the morphological formation are not well resolved at present. It has been suggested that the diluent molecules confined in the nano-scaled IL regions are deformed by the crystals and hence have a lower conformational entropy. ${ }^{7}$ An entropic driving force is thus developed, tending to pull diluent molecules out of the IL zones. This entropic force competes against the favorable interaction between the diluent and the amorphous portion of the crystalline polymer in the IL regions.

In addition to the thermodynamic driving force, the kinetic effect associated with the diluent mobility may also be a decisive factor. The competitive effect of diluent diffusivity $(D)$ and crystal growth rate $(G)$ has been proposed to govern the segregation distance. ${ }^{8-10}$ A small diluent diffusivity coupled with a fast crystal growth tend to trap the diluent into the IL regions. Such 
a competitive effect is represented by the morphological parameter, $\delta=D / G$, defined by Keith and Padden. ${ }^{10}$ The $\delta$ parameter has a length unit, so it could serve as an empirical measure for the segregation distance. However, using this parameter to predict, say, the composition and temperature effects on the morphological pattern is not straightforward; this is because that $G$ and $D$ are usually interdependent in the sense that the growth rate must depend on how fast the diluent molecules are removed away from the growth front.

As it is widely believed that the kinetic effect should surmount the thermodynamic driving force in the structural formation, several studies have critically examined the influence of diluent mobility on the morphology of C/A blends..$^{3,11-13}$ A summary of the results has recently been given by Debier et al. ${ }^{13}$ IL segregation was often observed when $T_{\mathrm{g}}$ of the diluent is similar to or higher than the crystallization temperature $\left(T_{\mathrm{c}}\right)$. When $T_{\mathrm{c}}$ became higher than $T_{\mathrm{g}}$ of the diluent, IF or IS segregation was observed. However, this simple rule was violated by some systems, implying that a much more complex mechanism controlled the morphological formation.

Among the various attempts in resolving the morphological controlling factor of C/A blends, the conclusions were often drawn through comparing the morphology of the blends of different polymers (e.g., the blends of a given crystalline polymer with various amorphous polymers with different $T_{\mathrm{g}} \mathrm{s}$ ). Very limited study has put forth to disclose the effects of composition, crystallization condition, and molecular weight on the morphological structure of a single system, which we believe is critical for identifying the controlling factor unambiguously. The reason why such a study is rare is probably due to the difficulty in resolving the subtle differences in morphological patterns caused by varying compositions and crystallization conditions. In the previous studies, we had explored the composition and $T_{\mathrm{c}}$ effects on the morphological structures of C/A blends based on the magnitude of volume fraction of lamellar stacks $\left(\phi_{\mathrm{S}}\right)$ measured by small angle X-Ray scattering (SAXS). ${ }^{4-6}$ This method has the disadvantage that the calculation of $\phi_{\mathrm{S}}$ relies largely on the determination of lamellar thickness from the one-dimensional correlation function $\left(\gamma_{1}(z)\right)$ or the interphase distribution function $\left(g_{1}(z)\right)$ whose shapes could be perturbed by the distribution of lamellar thickness. ${ }^{14,15}$ Moreover, the assignment of the nature of the layer thickness (i.e., the thickness of the crystalline or amorphous layers) determined from $\gamma_{1}(z)$ and $g_{1}(z)$ is sometimes ambiguous due to the Babinet reciprocity theorem. ${ }^{16}$

In the present study, we systematically investi- gate the dependence of morphology on composition and $T_{\mathrm{c}}$ for a melt-miscible poly(vinylidene fluoride) (PVDF)/poly(vinyl acetate) (PVAc) blend by means of SAXS. The absolute scattering intensity is measured here for calculating the scattering invariant in absolute unit. The invariant is proportional to the square of the electron density contrast between the crystalline and amorphous layers in the lamellar stacks. ${ }^{16}$ If the amorphous diluent resides in the IL regions, the electron density contrast will be modified accordingly and this will be manifested from the perturbation in invariant. Therefore, the composition and $T_{\mathrm{c}}$ effects can be revealed conveniently from the corresponding effects on scattering invariant. In this study, the morphological diagram of PVDF/PVAc will be constructed based on the observed extent of IL segregation at various compositions and $T_{\mathrm{c}} \mathrm{s}$. It will be shown that, even at a low $T_{\mathrm{c}}$ where the diluent mobility is expected to be sluggish, a noticeable portion of PVAc is expelled extralamellarly during the crystallization because of slow PVDF crystal growth. The results strongly suggest that crystal growth rate is more critical than the diluent mobility in controlling the structural formation in this binary system.

\section{EXPERIMENTAL}

\section{Materials an Blend Preparation}

PVDF with molecular weight of 60000 was purchased from Polysciences Inc. and PVAc with molecular weight of 80000 was acquired from Aldrich. Blendings of PVDF and PVAc were carried out by solution casting. The blending components were dissolved in $\mathrm{N}, \mathrm{N}$-dimethyl foramide (DMF) at room temperature yielding a $1 \mathrm{wt} \%$ solution. The solution was subsequently poured onto a petrie dish and the blend film was obtained after evaporating most solvent on a hot plate at $c a .80^{\circ} \mathrm{C}$. The blend film was further dried in vacuo at $60^{\circ} \mathrm{C}$ for $24 \mathrm{~h}$.

Specimens for SAXS study were prepared by pressing the blends between two pieces of Teflon ${ }^{\circledR}$ films on a Linkam HFS 91 hot stage at $190^{\circ} \mathrm{C} \pm 0.2^{\circ} \mathrm{C}$ for $3 \mathrm{~min}$, followed by quickly transferring the samples into an oven equilibrated at the desired crystallization temperatures $\left(T_{\mathrm{c}}=20\right.$ to $\left.160^{\circ} \mathrm{C}\right)$. The crystallizations were allowed to proceed for $72 \mathrm{~h}$. Characterization of the annealed samples by GPC and FT-IR indicated no sign of degradation through the thermal treatment.

\section{SAXS Measurement}

All SAXS experiments were conducted at room temperature. The X-Ray source, an $18 \mathrm{~kW}$ rotating anode X-Ray generator (Rigaku) equipped with a rotating anode $\mathrm{Cu}$ target, was operated at $200 \mathrm{~mA}$ and 40 
$\mathrm{kV}$. The incident X-Ray beam was monochromated by a pyrolytic graphite and a set of three pinhole inherent collimators were used so that the smearing effects inherent in slit-collimated small angle X-Ray cameras can be avoided. The scattering intensity was detected by a two-dimensional position sensitive detector (ORDELA Model 2201X, Oak Ridge Detector Laboratory Inc., U. S. A.) with $256 \times 256$ channels (active area $20 \times 20 \mathrm{~cm}^{2}$ with $\sim 1 \mathrm{~mm}$ resolution). The sample to detector distance was $2100 \mathrm{~mm}$ long. All data were corrected by the background (dark current and empty beam scattering) and the sensitivity of each pixel of the area detector. The data were radially (azimuthually) averaged in the $q$ range: $0.1 \mathrm{~nm}^{-1}<q<2.5 \mathrm{~nm}^{-1}$, ( $q=4 \pi / \lambda \sin (\theta / 2)$, where $\lambda$ is the $\mathrm{X}$-Ray wavelength and $\theta$ is the scattering angle), and converted to an absolute differential scattering cross section by means of pre-calibrated secondary standard. ${ }^{17}$ All the intensity profiles reported here had also been corrected for thermal diffuse scattering (TDS). The intensity level of TDS was assumed to be a constant and its magnitude was determined from the slope of $I q^{4} v s . q^{4}$ plot. $^{7}$

\section{Bulk Crystallinity Measurements}

Bulk crystallinities of crystalline PVDF/PVAc were calculated from the enthalpy of melting. The enthalpy of melting was measured by a TA Instrument 2000 differential scanning calorimeter (DSC). Bulk crystallinities were calculated by taking $104.5 \mathrm{~J} \mathrm{~g}^{-1}$ as the enthalpy of melting of $100 \%$ crystalline PVDF. ${ }^{18}$

\section{Crystal Growth Rate Measurement}

The spherulite growth was monitored with a Pac Hund polarized optical microscope. The sample was first melted on a Linkam HFS91 hot stage at $190^{\circ} \mathrm{C}$ for 3 min followed by quick transfer to another hot stage equilibrated at $T_{\mathrm{c}}$ where the spherulite growth was monitored. Micrographs were taken at intervals for measuring the spherulite radii $(R)$ as a function of time. The growth rate was calculated from the change of spherulite radius with time, $\mathrm{d} R / \mathrm{d} t$.

\section{RESULTS AND DISCUSSION}

The melt miscibility between PVDF and PVAc had already been verified previously where no sign of liquid-liquid phase separation was identified above or below the melting point. ${ }^{19-21}$ In the present study, the morphology generated through isothermal crystallizations at temperatures ranging from 20 to $160^{\circ} \mathrm{C}$ is probed by SAXS. Figure 1 displays the representative SAXS profiles of the blends crystallized at $T_{\mathrm{c}}=20,70$, 110 , and $160^{\circ} \mathrm{C}$. For all $T_{\mathrm{c}} \mathrm{s}$ investigated, the scatter-

ing intensity associated with the PVDF lamellar stacks grows upon blending with PVAc. The enhancement in scattering intensity, as found in a number of C/A systems, can be attributed to the incorporation of PVAc into the IL regions in that the electron density of PVAc $\left(0.637 \mathrm{~mol} \mathrm{e} \mathrm{cm}{ }^{-3}\right)$ is lower than that of amorphous PVDF $\left(0.833 \mathrm{~mol} \mathrm{e} \mathrm{cm}^{-3}\right)$ and its presence in the IL regions would inevitably enhance the electron density contrast between the crystalline and amorphous layers.

In addition to the perturbation in scattering intensity, the scattering peaks also shift toward lower $q$ upon blending indicating the long period $(L)$ of the lamellar stacks increases. The swelling in long period could arise from the swelling in amorphous layers caused by the IL segregation of PVAc. Figure 2 plots the long period against the weight fraction of PVAc $\left(w_{\text {PVAc }}\right)$. Depending upon the crystallization temperatures, the composition variation of $L$ is seen to exhibit different trends; $L$ increases monotonically with $w_{\text {PVAc }}$ for $T_{\mathrm{c}}=110$ and $160^{\circ} \mathrm{C}$ while it exhibits a maximum at $w_{\text {PVAc }} 0.2$ and levels off at $w_{\text {PVAc }} 0.3$ for $T_{\mathrm{c}}=20$ and $70^{\circ} \mathrm{C}$, respectively.

Elucidating the morphology from the perturbation of $L$ could be ambiguous because $L$ represents the sum of the crystalline and amorphous layer thickness. To reveal the morphological patterns accurately, the perturbation in scattering intensity is examined quantitatively through the determination of the invariant, $Q$, from the following well-known formula ${ }^{16}$

$$
Q=\frac{1}{2 \pi^{2}} \int_{0}^{\infty} I q^{2} \mathrm{~d} q
$$

Figure 3 plots $Q$ as a function of $w_{\mathrm{PVAc}}$. The composition dependence of $Q$ is analogous to that of long period. Except for $T_{\mathrm{c}}=110^{\circ} \mathrm{C}$ where $Q$ increases monotonically with $w_{\mathrm{PVAc}}$, the invariant is found to display a shallow maximum at $w_{\mathrm{PVAc}}=0.3$ or 0.2 . The reduction of $Q$ above these compositions could stem from the partial extralamellar segregation of PVAc; nevertheless, we have to be aware of the fact that the presence of crystal-amorphous interphase may also lead to a lower invariant compared with that prescribed by the ideal two-phase model. ${ }^{22}$ If the interphase only accounts for a minor portion in the system, the morphology of the semicrystalline polymer can be discussed conveniently in terms of the "corresponding two-phase model". 22 The invariant associated with this model, $Q_{\text {id }}$, is usually extrapolated from the measured value to eliminate the perturbation from the interphase. This involves the use of the unnormalized 1-D correlation function: ${ }^{22}$

$$
K(z)=\frac{1}{2 \pi^{2}} \int_{0}^{\infty} I q^{2} \cos q z \mathrm{~d} q
$$

$Q_{\text {id }}$ is obtained through the linear extrapolation from 

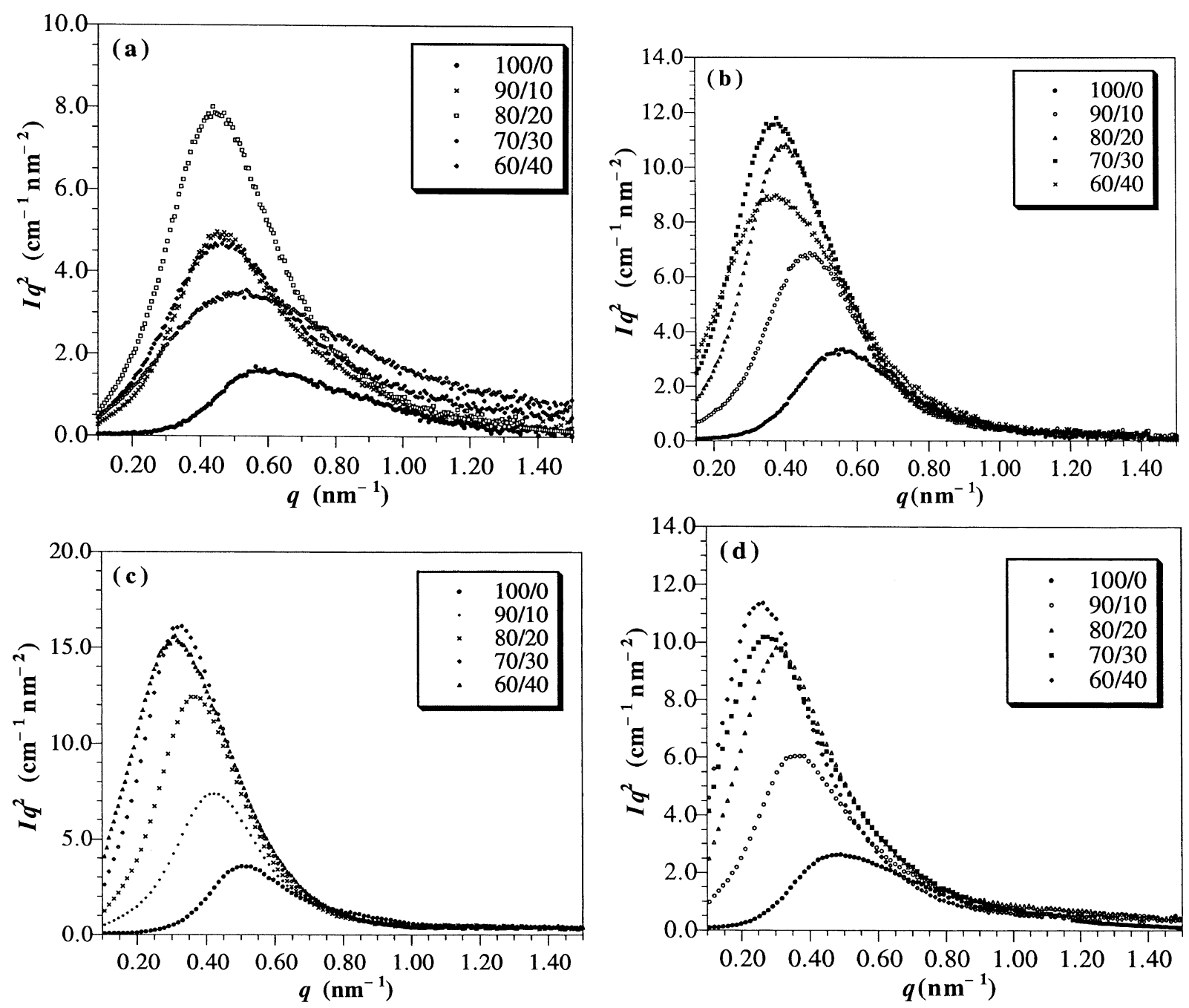

Figure 1. Lorentz-corrected SAXS profiles of PVDF/PVAc blends crystallized at (a) $20^{\circ} \mathrm{C}$, (b) $70^{\circ} \mathrm{C}$, (c) $110^{\circ} \mathrm{C}$, and (d) $160^{\circ} \mathrm{C}$.

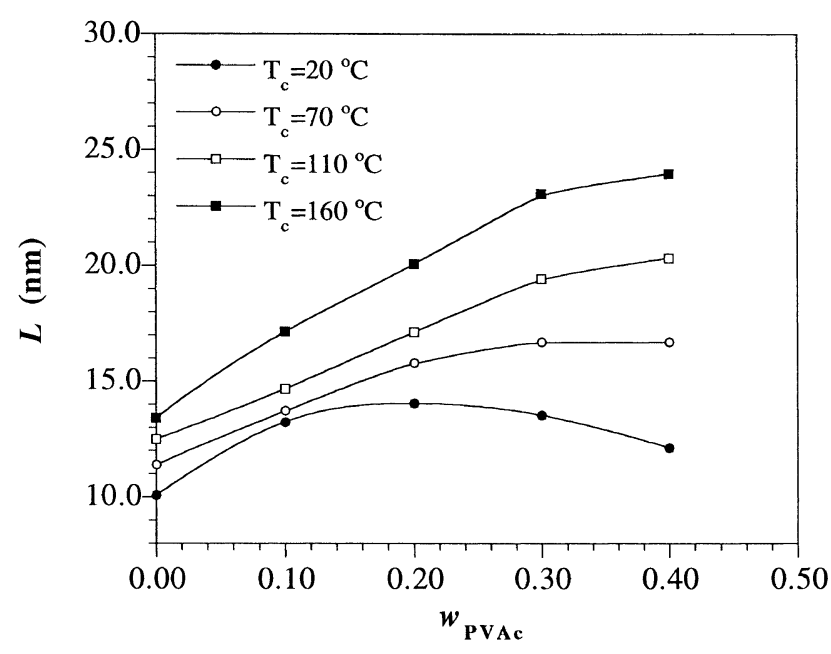

Figure 2. Composition variation of long period of PVDF/PVAc crystallized at various temperatures.

the self-correlation triangle of $K(z)$ to $z=0$ as demonstrated in Figure 4.

Figure 5 plots the observed $Q_{\mathrm{id}}$ as a function of $w_{\text {PVAc }}$. The composition dependence of $Q_{\text {id }}$ is virtually the same as that of $Q$. To reveal the morphology

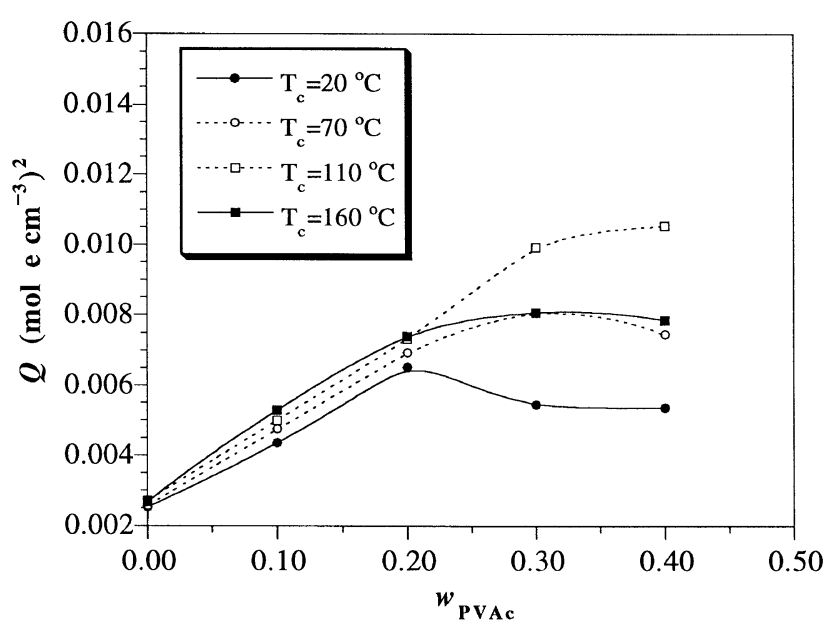

Figure 3. Composition dependence of the SAXS invariant determined directly from the scattering profiles via eq 1 .

in terms of the extent of IL segregation, we now compare the observed $Q_{\text {id }}$ with the invariant, $Q_{\mathrm{IL}}$, calculated by assuming that all PVAc is located in the IL regions upon PVDF crystallization. $Q_{\text {id }}$ should be smaller than $Q_{\mathrm{IL}}$ in the case where a portion of PVAc is located 


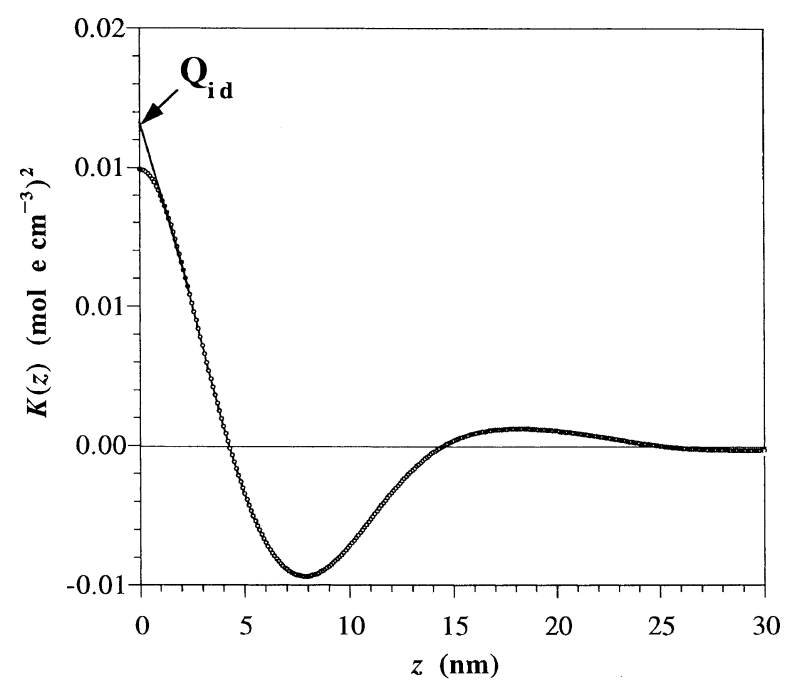

Figure 4. Representative plot of the Strobl-Schneider's onedimensional correlation function. The invariant associated with the corresponding two-phase model, $Q_{\text {id }}$, is extrapolated from the selfcorrelation triangle as shown in the figure.

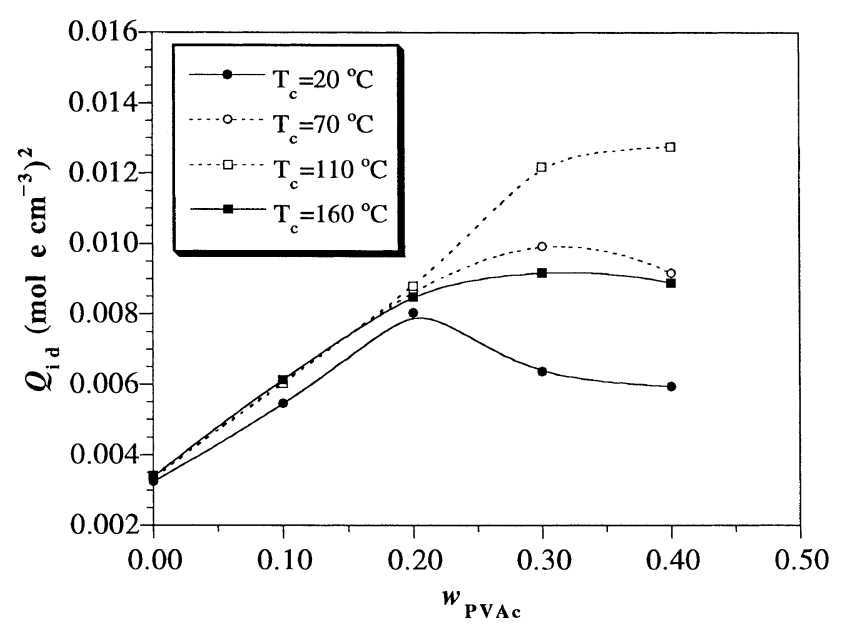

Figure 5. Composition variation of $Q_{\text {id }}$ of PVDF/PVAc crystallized at various $T_{\mathrm{c}} \mathrm{s}$.

extralamellarly. $Q_{\mathrm{IL}}$ corresponds to the invariant of a two-phase system homogeneously filled with lamellar stacks, which is given by

$$
Q_{\mathrm{IL}}=\phi_{\mathrm{c}}\left(1-\phi_{\mathrm{c}}\right) \eta^{2}
$$

where $\phi_{\mathrm{c}}$ is the bulk crystallinity and $\eta$ is the electron density contrast between PVDF crystal and the IL amorphous phase. $\eta$ can be calculated by

$$
\eta=\phi_{\mathrm{PVAc}}{ }^{\mathrm{IL}} \eta_{\mathrm{PVAc}}+\left(1-\phi_{\mathrm{PVDF}}{ }^{\mathrm{IL}}\right) \eta_{\mathrm{PVDF}}
$$

where $\phi_{\mathrm{PVAc}}{ }^{\mathrm{IL}}$ is the volume fraction of PVAc in the IL regions, $\eta_{\mathrm{PVAc}}=\eta_{\mathrm{PVDF}}{ }^{\mathrm{c}}-\eta_{\mathrm{PVAc}}=0.318 \mathrm{~mol} \mathrm{e} \mathrm{cm}^{-3}$, the electron density contrast between crystalline PVDF and PVAc; and $\eta_{\mathrm{PVDF}}=\eta_{\mathrm{PVDF}}{ }^{\mathrm{c}}-\eta_{\mathrm{PVDF}}{ }^{\mathrm{a}}=0.121 \mathrm{~mol} \mathrm{e}$ $\mathrm{cm}^{-3}$, the contrast between crystalline and amorphous PVDF. Assuming a complete IL segregation of PVAc, the weight fraction of PVAc in the IL regions can be

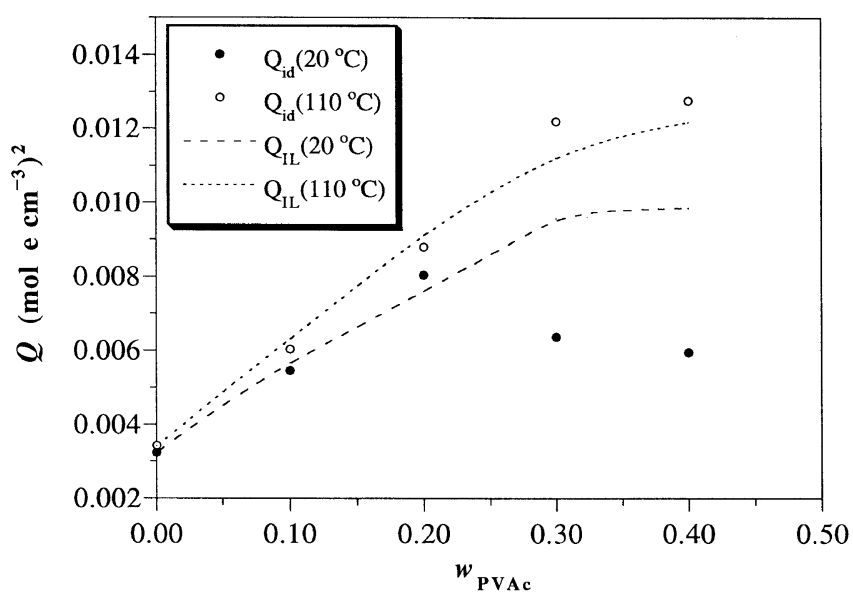

Figure 6. Comparison between the observed $Q_{\text {id }}$ and the invariant, $Q_{\mathrm{IL}}$, calculated by assuming complete IL segregation for $\mathrm{PVDF} / \mathrm{PVAc}$ crystallized at 20 and $110^{\circ} \mathrm{C}$. The dashed lines signify the composition variation of the calculated $Q_{\mathrm{IL}}$.

easily obtained with the knowledge of crystallinity, viz.

$$
w_{\mathrm{PVAc}}{ }^{\mathrm{IL}}=\frac{w_{\mathrm{PVAc}}{ }^{0}}{1-w_{\mathrm{c}}}
$$

where $w_{\text {PVAc }}{ }^{0}$ is the overall weight fraction of PVAc in the blend and $w_{\mathrm{c}}$ is the weight fraction of PVDF crystals. After converting $w_{\mathrm{PVAc}} \mathrm{IL}^{\mathrm{IL}}$ into the volume fraction $\left(\phi_{\mathrm{PVAc}}{ }^{\mathrm{IL}}\right)$, the expected invariant for a full IL segregation can be calculated accordingly.

Figure 6 compares $Q_{\text {id }}$ with the corresponding $Q_{\mathrm{IL}}$ for the blends crystallized at 110 and $20^{\circ} \mathrm{C}$. For $T_{\mathrm{c}}=$ $110^{\circ} \mathrm{C}$, the observed $Q_{\text {id }}$ always agrees within $8 \%$ with the calculated $Q_{\mathrm{IL}}$ over the composition range investigated, suggesting that nearly all PVAc was present in the IL regions after PVDF crystallization. In the case where crystallization was conducted at a much lower $T_{\mathrm{c}}$ of $20^{\circ} \mathrm{C}$, the observed $Q_{\text {id }}$ is more than $30 \%$ lower than the corresponding $Q_{\mathrm{IL}}$ when the PVAc composition exceeds $20 \mathrm{wt} \%$. This strong negative deviation indicates that a noticeable portion of PVAc is located exterior to the IL regions.

The comparison between $Q_{\mathrm{id}}$ and $Q_{\mathrm{IL}}$ can be reduced to a single morphological index, $\alpha$, defined by the ratio of the two quantities, i.e., $\alpha=Q_{\mathrm{id}} / Q_{\mathrm{IL}}$. In the case of full IL segregation $\alpha=1$ and it decreases with increasing extent of extralamellar segregation. Figure 7 shows the composition variation of $\alpha$ for the blends crystallized at various $T_{\mathrm{c}} \mathrm{s}$. Large negative deviation (>10\%) from unity becomes prevalent when the PVAc composition exceeds $20 \mathrm{wt} \%$, showing that increasing PVAc composition tends to promote extralamellar segregation or the distance of diluent segregation. This effect has also been identified previously in other C/A systems containing an amorphous diluent with a higher $T_{\mathrm{g}} .{ }^{5,6}$

Based on the results of Figure 7, a morphological diagram of PVDF/PVAc is constructed in Figure 8 so that 


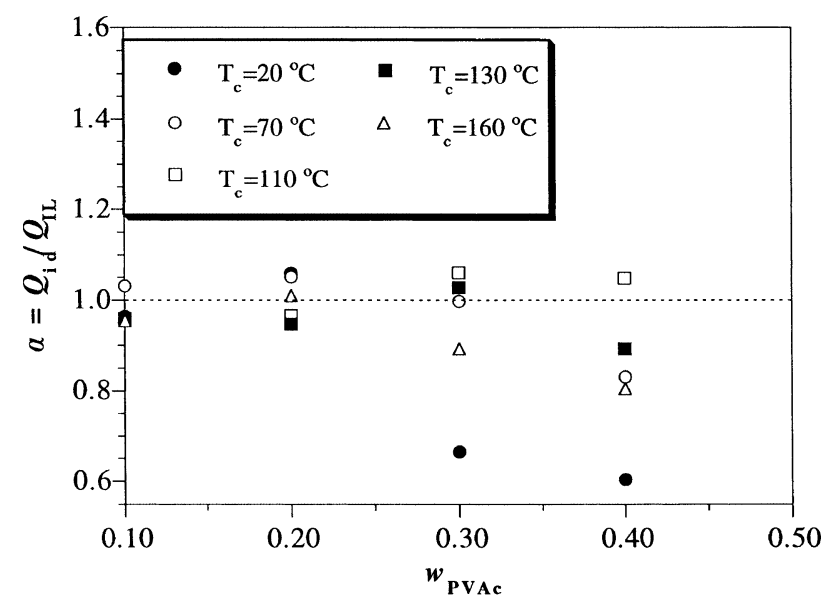

Figure 7. Composition dependence of the morphological index, $\alpha=Q_{\mathrm{id}} / Q_{\mathrm{IL}}$, for PVDF/PVAc crystallized at various $T_{\mathrm{c}} \mathrm{s}$.

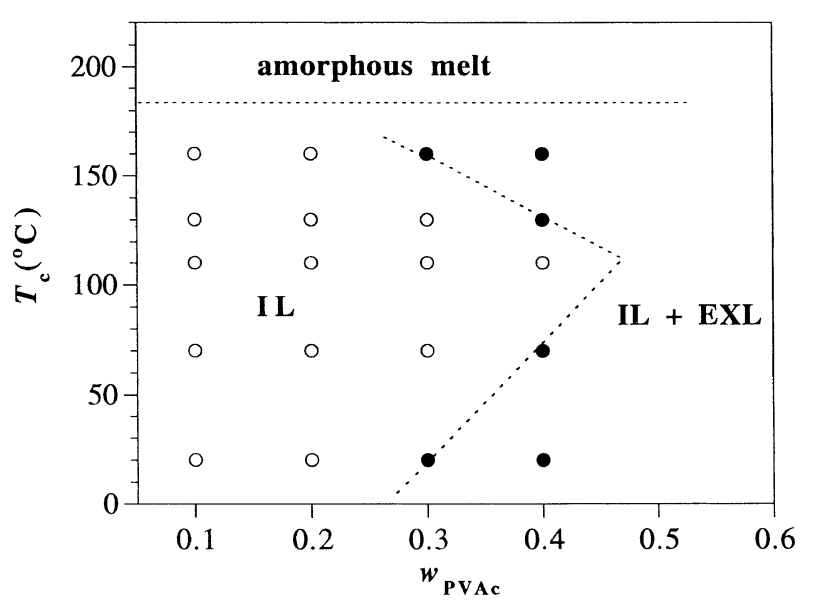

Figure 8. The morphological diagram of PVDF/PVAc blends. The left region enclosed by the dashed line signifies the $T_{\mathrm{c}}$ and composition range wherein the diluent segregation is predominantly interlamellar. The right region represents the coexistence of IL and extralamellar segregation.

the effects of composition and $T_{\mathrm{c}}$ can be clearly identified. The left region enclosed by the dashed lines signifies the $T_{\mathrm{c}}$ and composition range wherein the morphology is predominantly interlamellar. It is seen that the extent of extralamellar segregation is minimized by crystallization at an intermediate $T_{\mathrm{c}}$ of $110^{\circ} \mathrm{C}$, while beyond certain compositions ( $w_{\mathrm{PVAc}}>0.2$ or 0.3 ) the crystallizations at either higher or lower $T_{\mathrm{c}} \mathrm{s}$ tend to draw a portion of PVAc out of the IL regions. Even crystallization at as low as $20^{\circ} \mathrm{C}$ (where the diluent mobility is expected to be sluggish) can result in a large extent of extralamellar segregation (the extent of extralamellar segregation is indeed the highest compared with that found at other $T_{\mathrm{c}} \mathrm{s}$ ). This result quickly rules out the possibility that the segregation distance is controlled by the diluent mobility in that extralamellar segregation should have been frustrated by decreasing temperature if the diluent mobility dominated the structural formation.

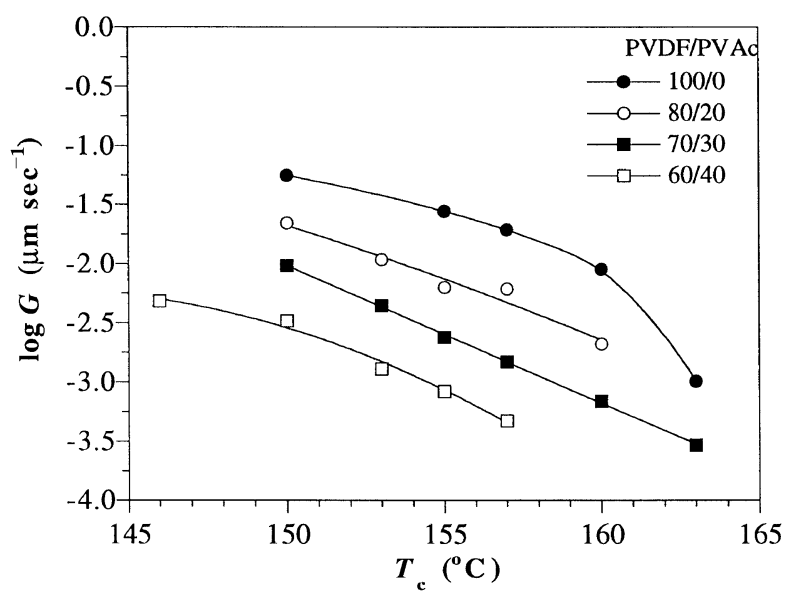

Figure 9. The crystal growth rates of PVDF/PVAc blends at $T_{\mathrm{c}} \mathrm{s}$ ranging from 146 to $163^{\circ} \mathrm{C}$.

Since diluent mobility does not closely control the segregation distance, we now proceed to consider whether another kinetic parameter, the crystal growth rate, is the governing factor. Figure 8 shows that the extent of extralamellar segregation displays a minimum at $T_{\mathrm{c}}=110^{\circ} \mathrm{C}$. This actually reconciles with the $T_{\mathrm{c}}$ dependence of crystal growth rate which usually exhibits a maximum at an intermediate $T_{\mathrm{c}}$ due to the interplay between the chain mobility and the driving force of crystallization. Since a fast crystal growth tends to prevent the diluent from escaping into the extralamellar regions, we expect the minimum extent of extralamellar segregation to be located at the $T_{\mathrm{c}}$ corresponding to the fastest crystal growth for a given blend composition, namely, at $T_{\mathrm{c}}=110^{\circ} \mathrm{C}$ for the present PVDF/PVAc systems. Figure 9 displays the measured crystal growth rates as a function of $T_{\mathrm{c}}$. The growth rates could only be measured over a limited $T_{\mathrm{c}}$ range from 146 to $163^{\circ} \mathrm{C}$ because the nucleation density below $146^{\circ} \mathrm{C}$ became too high to allow the accurate resolution of spherulite growth. Nevertheless, the available growth data can be analyzed by the Lauritzen-Hoffman theory ${ }^{23,24}$ and the results can be applied subsequently to calculate the growth rates over the entire crystallization envelop. The Lauritzen-Hoffman's equation of crystal growth rate reads

$$
G=G_{0} \exp \left[-\frac{U^{*}}{R\left(T_{\mathrm{c}}-T_{0}\right)}\right] \exp \left[-\frac{K_{\mathrm{g}}}{T_{\mathrm{c}} \Delta T f}\right]
$$

where $G_{0}$ is the pre-exponential factor; $U^{*}$ is the activation energy of segmental transport; $T_{0}$ is the temperature at which such a transport ceases; $T=\left(T_{\mathrm{m}}{ }^{0}-T_{\mathrm{c}}\right)$, the degree of undercooling; $f=2 T_{\mathrm{c}} /\left(T_{\mathrm{m}}{ }^{0}+T_{\mathrm{c}}\right)$, a factor taking account of the temperature dependence of the enthalpy of melting; and $K_{\mathrm{g}}$ is a nucleation constant depending upon the surface free energies of the secondary nuclei. Equation 6 prescribes that a straight line with intercept and slope of $\ln G_{0}$ and $-K_{\mathrm{g}}$, respectively, 


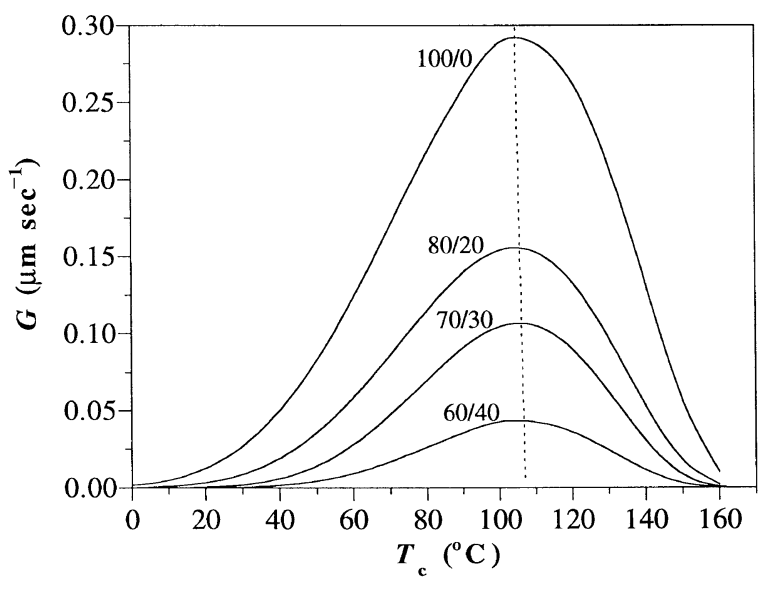

Figure 10. The crystal growth rates of PVDF/PVAc blends calculated by the Lauritzen-Hoffman theory. It can be seen that the maximum growth rates occur in the vicinity of $110^{\circ} \mathrm{C}$ where the systems display the maximum extent of IL segregation.

should be obtained in the plot of $\ln G+U^{*} / R\left(T_{\mathrm{c}}-T_{0}\right)$ vs. $1 / T_{\mathrm{c}}$ Tf. Using the available data of $G$ and the typical values of $U^{*}=1500 \mathrm{cal} \mathrm{mol}^{-1}$ and $T_{0}=T_{\mathrm{g}}-30,{ }^{24}$ the plots were generated for PVDF/PVAc blends. The values of $G_{0}$ and $K_{\mathrm{g}}$ thus determined were then substituted into eq 6 to calculate the crystal growth rates over the entire crystallization envelop. The results are presented in Figure 10. For all compositions investigated, the growth rates are seen to exhibit a maximum near $110^{\circ} \mathrm{C}$ where the extralamellar segregation is the mostly frustrated. This strongly corroborates the postulate that PVAc segregation is predominantly controlled by the crystal growth rate.

Although intuitively the segregation distance should be intimately connected with the diluent mobility, the present study demonstrates that crystal growth rate plays a more important role in PVDF/PVAc system. Similar conclusions have been drawn by Tlibuddin et $a l$. in the study of strongly interacting C/A blends ${ }^{3}$ and also our previous SAXS investigations (with relative intensity measurement) on the systems crystallized over a limited $T_{\mathrm{c}}$ range. ${ }^{5,6}$

\section{CONCLUSIONS}

The effect of crystallization temperature on the morphological patterns of PVDF/PVAc has been investigated to reveal the governing factor of structural formation in the system. IL segregation of PVAc was always observed over the composition and $T_{\mathrm{c}}$ range investigated but the extent of IL segregation depended on both parameters. The extent of IL segregation did not increase monotonically with decreasing $T_{\mathrm{c}}$ but exhibited a maximum at an intermediate $T_{\mathrm{c}}$ of $110^{\circ} \mathrm{C}$, indicating that the segregation distance was not dominated by the diluent mobility. Excellent correlations were found between the extent of IL segregation and the growth rate which strongly suggested that the growth kinetics predominantly controlled the average distance of diluent segregation.

Acknowledgment. This work is supported by the National Science Council, R. O.C. under grant NSC 89-2216-E-007-055.

\section{REFERENCES}

1. R. S. Stein, F. B. Khambatta, F. P. Warner, T. Russell, A. Escala, and E. Balizer, J. Polym. Sci., Polym. Symp., 63, 313 (1978).

2. M. Vanneste, G. Groeninckx, and H. Reynaers, Polymer, 38, 4407 (1997).

3. S. Talibuddin, L. Wu, J. Runt, and J. S. Lin, Macromolecules, 29, 7527 (1996).

4. H.-L. Chen, L.-J. Li, and T.-L. Lin, Macromolecules, 31, 2255 (1998).

5. H.-L. Chen, S.-F. Wang, and T.-L. Lin, Macromolecules, 31, 8924 (1998).

6. H.-J. Chiu, H.-L. Chen, T.-L. Lin, and J. S. Lin, Macromolecules, 32, 4969 (1999).

7. T. P. Russell, H. Ito, and G. D. Wignall, Macromolecules, 21, 1703 (1988).

8. H. D. Keith and F. J. Padden, J. Polym. Sci, 51, 84 (1961).

9. H. D. Keith, J. Polym. Sci., Part A: Polym. Chem., 2, 4339 (1964).

10. H. D. Keith and F. J. Padden, J. Appl. Phys., 35, 127 (1975).

11. G. Defieuw, G. Groeninckx, and H. Reynaers, Polym. Commun., 30, 267 (1989).

12. T. Okada, H. Saito, and T. Inoue, Polymer, 35, 5699 (1994).

13. D. Debier, A. M. Jonas, and R. Legras, J. Polym. Sci., Part B: Polym. Phys., 36, 2197 (1998).

14. C. G. Vonk and G. Kortleve, Kolloid Z. Z. Polym., 220, 19 (1967).

15. C. S. Santa Cruz, N. Stribeck, H. G. Zachmann, and F. J. Balta' Calleja, Macromolecules, 24, 5980 (1991).

16. R.-J. Roe, "Methods of X-Ray and Neutron Scattering in Polymer Science", Oxford University Press, Inc., London, $2000, \mathrm{p} 31$.

17. T. P. Russell, J. S. Lin, S. Spooner, and G. D. Wignall, J. Appl. Crystallogr., 21, 629 (1988).

18. U. Gaur, B. B. Wunderlich, and B. Wunderlich, J. Phys. Chem. Ref. Data, 12, 29 (1983).

19. D. R. Paul, J. W. Barlow, R. E. Bernstein, and D. C. Wahrmund, J. Polym. Sci., Polym. Phys. Ed., 18, 1225 (1978).

20. D. R. Paul and J. W. Barlow, J. Macromol. Sci., Rev. Macromol. Chem., C18, 109 (1980).

21. Y. Okabe, T. Kyu, H. Saito, and T. Inoue, Macromolecules, 31, 5823 (1998).

22. G. R. Strobl and M. Schneider, J. Polym. Sci., Polym. Phys. $E d ., 18,1343$ (1980).

23. J. D. Hoffman and J. J. Weeks, J. Res. Natl. Bur. Stand., A Phys. Chem., 66A, 13 (1962).

24. J. D. Hoffman and R. L. Miller, Polymer, 38, 3151 (1997). 